University of Nebraska - Lincoln

DigitalCommons@University of Nebraska - Lincoln

\title{
Estimating Dilemma Zone Hazard Function at High Speed Isolated Intersection
}

\author{
Anuj Sharma \\ University of Nebraska at Lincoln, anujs@iastate.edu \\ Darcy Bullock \\ Purdue University \\ Srinivas Peeta \\ Purdue University
}

Follow this and additional works at: https://digitalcommons.unl.edu/civilengsharma

Part of the Civil Engineering Commons

Sharma, Anuj; Bullock, Darcy; and Peeta, Srinivas, "Estimating Dilemma Zone Hazard Function at High Speed Isolated Intersection" (2010). Anuj Sharma Publications. 2.

https://digitalcommons.unl.edu/civilengsharma/2

This Article is brought to you for free and open access by the Civil Engineering at DigitalCommons@University of Nebraska - Lincoln. It has been accepted for inclusion in Anuj Sharma Publications by an authorized administrator of DigitalCommons@University of Nebraska - Lincoln. 
Published in Transportation Research Part C: Emerging Technologies (2010); doi: 10.1016/j.trc.2010.05.002

Published by Elsevier Ltd. Used by permission.

Submitted August 6, 2008; revised May 6, 2010; accepted May 12, 2010; published online June 29, 2010.

\title{
Estimating dilemma zone hazard function at high speed isolated intersection
}

\author{
Anuj Sharma \\ Department of Civil Engineering, University of Nebraska-Lincoln, \\ 330F Whittier Building, Lincoln, NE 68583-0851, USA \\ Darcy Bullock and Srinivas Peeta \\ School of Civil Engineering, Purdue University, West Lafayette, Indiana, USA \\ Corresponding author - A. Sharma, tel 402 472-6391, fax 402 472-0859, email asharma@unl.edu
}

\begin{abstract}
The traditional surrogate measures of safety (like number of vehicles in dilemma zone) fail to quantify the risk of crash. Traffic conflict has been proposed as an improved surrogate measure of safety for operations at isolated intersections to quantify the risk. This paper develops a dilemma zone hazard function estimating procedure to obtain the probability of traffic conflict occurring. This approach is an extension of the current approach of dilemma zone boundaries to determine the risk of traffic conflict for an individual vehicle in the case of a dilemma zone incursion. Field data collected from the intersection of SR37 and SR32 at Noblesville, Indiana is used to generate a binary choice model that best explains the underlying criteria for a driver's decision at the onset of yellow. The probability of making an erroneous decision is used as the probability of traffic conflict (dilemma hazard function). An economic framework was developed to implement the dynamic of dilemma hazard function using existing controllers. Although the data are specific to one intersection, the procedures are readily transferable. This paper also demonstrates the potential of sensor providing richer data than an inductive loop detector can be used to further enhance the safety at signal operations.
\end{abstract}

Keywords: dilemma zone hazard, driver's decision models, traffic signal operations, traffic conflict, perceived conflict

\section{Introduction}

Green extension systems are deployed at rural high speed signalized intersections to reduce the number of red light violations and rear end crashes. The primary objective of these systems is to minimize the occurrence of high speed vehicles in a dilemma zone. The green phase of the high speed approach is extended until there is no vehicle in the dilemma zone. An upper threshold, maximum green time, is provided for this operation to avoid excessive delays to the cross street traffic. This approach is an all-or-nothing approach. High speed vehicles are provided complete protection against dilemma zone incursions before the maximum green is reached. However, if the maximum green is reached, the protection is completely withdrawn. Consequently, there exist no intermediate levels of protection; the signal logic either provides a $100 \%$ protection against dilemma zone in case of gap out or the protection drops to $0 \%$ in case of max out. This, simultaneous gap out, logic works well under low volume conditions where there is a high chance of finding a duration when no vehicles are in there dilemma zone prior to max-out. But probability of max out increase substantially during medium to high volumes. A detailed analysis of this problem has been described by Sharma et al. (2006). It was shown that the implementation of the simultaneous gap-out logic at an intersection in Noblesville, Indiana led to max-out as high as $40 \%$ of cycles per hour during peak hours and around 200 dilemma zone incursions per day. 
Sharma et al. (2007) developed a marginal cost and benefits approach to address shortcoming of the simultaneous gap-out logic and competing models. In this paper, a methodology was proposed to gradually remove the protection one lane at a time as the maximum green time approaches. Based on this approach, the high-speed intersection can be operated in an economically efficient manner using the following control logic:

(1) A minimum green time is allotted to each phase for avoiding "short-green" dilemma. Parsonson (1978) suggested this term for the scenario when the green is too short to violate driver's expectancy. Minimum green time may also be governed by pedestrian safety issues.

(2) Phases remain green beyond their minimum green as long as they are still discharging at or near saturation (unless the maximum green time is reached.) This is implemented using stop bar detectors (Smaglik et al., 2005).

(3) All the lanes in both directions of high speed through phase are protected until the cost experienced by the opposing movements exceeds the estimated safety benefits associated with extending the phase. After this point one vehicle is allowed to be in the dilemma zone at the termination of green. This approach continues to drop one lane at a time till max-green time is reached. This allows the problem to be cast as a marginal costs-benefits problem.

Sharma et al. (2007) proposed to use the probability of traffic conflict to estimate the safety benefits but did not provide a model for measuring it. This paper develops a model to derive the probability of traffic conflict termed as dilemma hazard function. Then the paper modifies the previous marginal cost and benefit algorithm to gradually shrink the dilemma zone boundaries for all lanes instead of removing it completely from a single lane. The modified signal control scheme will dynamically vary vehicle extensions based on the opposing queue lengths and passage of green time. This modified signal control scheme can be implemented at any intersection having an advance speed trap to measure the speeds of the approaching vehicle.

\section{Surrogate measures of safety}

Typically, the total number of vehicles in the dilemma zone has been used as a surrogate measure for safety at rural high-speed intersections. Dilemma zone was initially defined as the area where the driver can neither stop comfortably nor clear safely on the onset of yellow. This approach (Gazis et al., 1960; May, 1968) uses deterministic design values such as perception reaction time, comfortable deceleration rate, length of yellow interval etc. to determine the location of dilemma zone. The stopping and clearing distance for a vehicle are calculated. Stopping distance $\left(X_{s}\right)$ is a distance from the stop bar prior to which any vehicle can stop using a comfortable deceleration. Clearing distance $\left(X_{c}\right)$ is a distance from stop bar after which any vehicle can cross the stop line using comfortable acceleration. The signal was operated such as to eliminate the existence of dilemma zone.

There can be three possible scenarios based on the values of stopping distance and clearing distance.

- $X_{s}>X_{c}$ : There exists a dilemma zone $\left(X_{s}>X>X_{c}\right)$ where a driver can neither stop comfortably nor clear safely.

- $X_{s}=X_{c}$ : There exists no dilemma zone.

- $X_{s}<X_{c}$ : There exists an option zone $\left(X_{s}<X<X_{c}\right)$ where a driver can both stop comfortably as well as proceed safely.

The main limitation of this approach is it assumes a perfect knowledge of all variables. In reality, drivers have a perception of various variables like distance from the stop bar, yellow duration etc. but lack perfect knowledge. The other limitation of this approach is that there is no conversion metric that can convert the presence of a vehicle in its dilemma zone to the magnitude of risk of crash. As a result this surrogate measure could not effectively be used to compare the tradeoff between safety and efficiency of operation at an intersection.

Researchers re-characterized the surrogate measure as decision dilemma zone to take into account the variability in human perception (Sheffi and Mahmassani, 1981). This decision dilemma zone is defined as that approach area within which the probability of deciding to stop on the display of yellow is within the range of $10-90 \%$. This zone is considered to have a higher risk for rear end collisions and red light violations as the driver is not sure whether to proceed through the intersection or to attempt to stop.

There have been several attempts to ascertain the dilemma zone boundaries (Olson and Rothery, 1962; Webster and Ellson, 1965; ITE technical committee, 1974; Zegeer, 1977; Sheffi and Mahmassani, 1981; Chang et al., 1985; Bonneson et al., 1994). Initially, a frequency based approach was used to obtain the probability of stopping. The percentage of drivers stopping for a given distance and speed was used develop the cumulative distribution function. Researchers observed a significant variation in the dilemma zone boundaries obtained from frequency based methods.

Binary discrete choice models were subsequently proposed to determine the probability of stopping for a given distance and speed for better understanding of the underlying human decision models and explain the variation in the observed dilemma zone boundaries (Sheffi and Mahmassani, 1981; Chang et al., 1985; Gates et al., 2007; Papaioannou, 2007).

There are common limitations with all of the above approaches. Although the dilemma zone boundaries were determined using a stochastic model, the definition of dilemma zone boundaries was still deterministic. The definition implies a driver in the area where the probability of stopping ranges from $10 \%$ to $90 \%$ is considered to be unsafe and 
anyone outside this area is considered to be safe. This binary approach states that a person is either at risk or risk free, but no comments are made about the level of risk at each location. Figure 1 contrasts the present surrogate measure of safety with the proposed surrogate measure of safety. The traditional green extension systems only mark the region of risk but do not quantify the varying levels of risk associated within the region (shown in Figure 1a). The green termination systems use the number of vehicles in the dilemma zone as a surrogate measure for quantifying the cost of risk. The number of vehicles is a rank-ordered metric (shown in Figure 1b) where the cost of one vehicle in a dilemma zone is less than the cost of two vehicles in a dilemma zone; but the cost is independent of the positions of vehicles in dilemma zone. This paper develops the probability of having a traffic conflict as an improved surrogate measure of safety to quantify the risk (shown in Figure 1c).

\section{Traffic conflict on the onset of yellow}

A preliminary order of events in driving is shown in Figure 2. A driver makes a decision based on his/her information about the system, ambient conditions, driver behavior, and vehicle type. Let us assume a driver observes a yellow light at time $t$ and decides to stop or go at time $t+\Delta t_{1}$. An erroneous decision could be made at time $t+\Delta t_{1}$ due to an error in the driver's perception of the surroundings. If the driver realizes his/her error after receiving feedback from the surrounding system, it is called a "detected error." If the driver detects the error, he/she then tries to rectify the error by taking an evasive action at time $t+\Delta t_{1}+\Delta t_{2}$. If the evasive action is successful, the normal traffic continuation is resumed. However, an unsuccessful evasive action results in a crash or traffic violation. The probability of a traffic conflict, therefore, will be the same as the probability of having a "detected error."

Single vehicle conflicts are defined as the conflicts when there is only one vehicle present in a lane in close vicinity of the intersection on the onset of yellow. An example of single vehicle conflict would be, a single vehicle after the onset of yellow suddenly realizes that he/she cannot safely make it through the intersection and applies heavy deceleration to stop (in absence of any other vehicle in the same lane). Multiple vehicle conflicts are defined as the conflicts

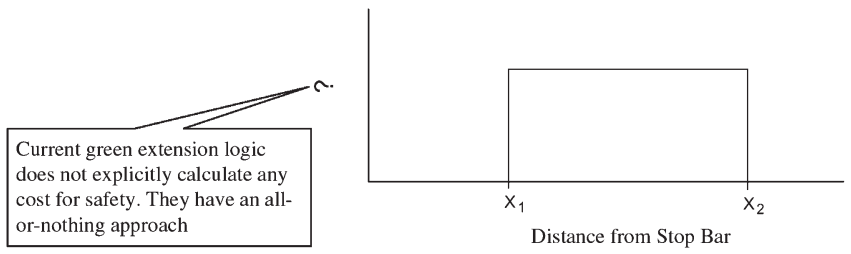

(a) Safety cost evaluation in current green extension systems.

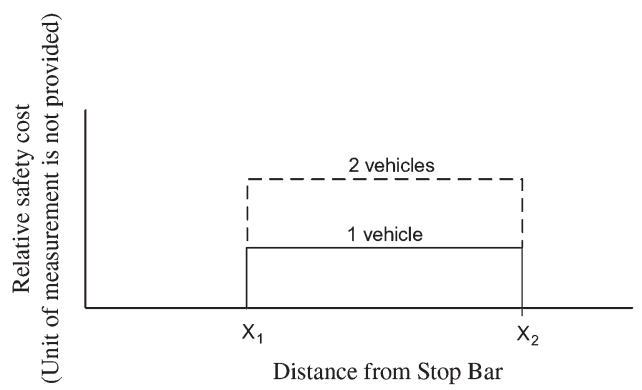

(b) Safety cost evaluation in advanced green termination systems.

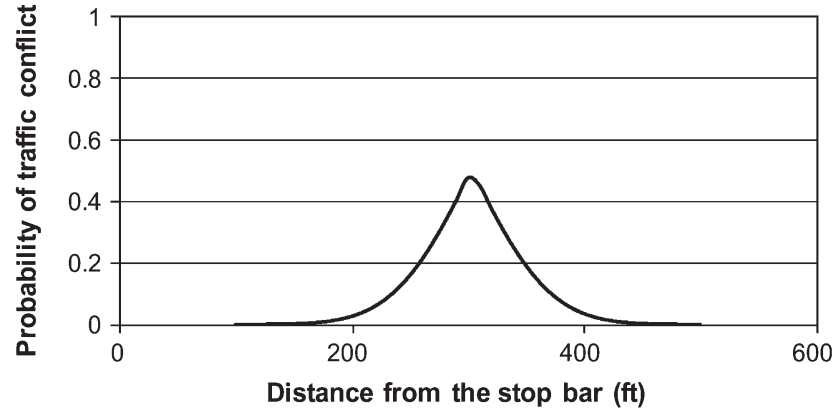

(c) Proposed evaluation of safety cost

Figure 1. Comparison of traditional and proposed surrogate methods for estimating safety. 


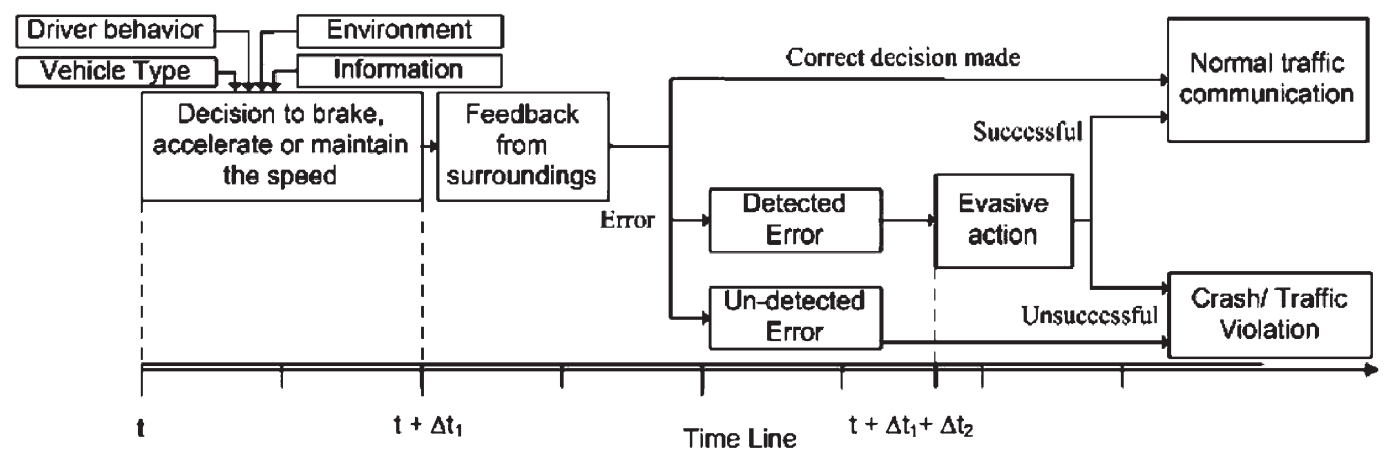

Figure 2. Driver decision process on the onset of yellow.

when there is more than one vehicle present in a lane in close vicinity of the intersection on the onset of yellow. An example of multiple vehicles conflict would be, if a vehicle closely following a lead vehicle swerves to avoid collision when lead vehicle suddenly decides to stop on the onset of yellow. In case of multiple vehicles, the decision model of each driver can become complicated as the decision of one driver affects the other and the correlated perception errors of both drivers will lead to complex error models.

In this paper, we develop a methodology to determine the dilemma hazard function for a single vehicle conflict. Empirical data is used to develop such a function for cars under normal weather and daytime conditions. A highspeed intersection of State Road (SR) 37 and State Road (SR) 32 in Noblesville, Indiana was used as the study site. Field data was used to generate binary choice model that best explains the underlying criteria for driver's decision on the onset of yellow. The probability of making an erroneous decision is used as the probability of traffic conflict. Then the paper develops a marginal cost and benefit signal control logic using a dynamic vehicle extension.

\section{Field data collection and validation}

The data collection site at the signalized intersection of SR 37 and SR 32 in Noblesville, Indiana is shown in Figure 3a. This instrumented intersection logs individual vehicle tracks and signal states. A simultaneous video of the existing traffic conditions is recorded for manual ground truth. Figure $3 \mathrm{~b}$ illustrates the data collection environment used for the evaluation.

The equipments used for data collection include:

- Southbound (SB) Wide Area Detector (WAD): SmartSensor Advance by Wavetronix was used as the WAD. This sensor uses a patented digital wave radar technology to track all the vehicles with a stated accuracy of $5 \mathrm{ft}$. The SB WAD was mounted on a mast arm $155 \mathrm{ft}$. behind the stop bar at a height of $32 \mathrm{ft}$.

- SB Video: A video camera mounted on the SB mast arm was used for visual validation. The video had capability to overlay signal actuations over the video captured by the video sensor.

This setup was used to estimate the speed and position of the vehicle entering the SB approach. Vehicles up to a distance of $450 \mathrm{ft}$ were detected by the WAD, and the WAD track files with the distance, speed, and identification numbers of the reported detections were logged. Also, the video output and the WAD output were displayed on a PC and were recorded by a screen capture device at a rate of 30 frames per second as shown in Figure $3 b$.

After installation of the WAD, it was validated against a handheld GPS device. An example of vehicle tracking by GPS and WAD is shown in Figure 4. A detailed analysis of the performance of the WAD can be found elsewhere (Sharma et al., 2008).

Data was collected on the southbound approach of SR37 starting September 2007-April 2008 from 6:00 AM to 8:00 PM. The video recorded, with WAD and video input, was analyzed manually to reduce 102 days of data. The data were collected during good weather conditions and in the absence of any special event. The time of the onset of yellow, the vehicle id number, driver's decision to stop or go, the vehicle velocity and the distance from the stop bar as given by the WAD, was noted. Only cases having one car in a lane at the onset of yellow were kept for analysis of a single vehicle conflict. Also, instances where the WAD performed erratically were deleted to maintain a higher accuracy. A total of 2,870 cars were observed (2219 Stop and $651 \mathrm{Go}$ ). The peak hour through volume on the two lanes of south bound approach varied between 600-900 vehicles per hour.

\section{Econometric modeling to gain insights in the driver's decision model}

\subsection{Best specified model parameters}

At the onset of yellow, a driver can choose from two mutually exclusive course of action: Stop or Go. The decision process thus can be modeled by binary discrete choice models. Based on the approach followed by Sheffi and Mah- 


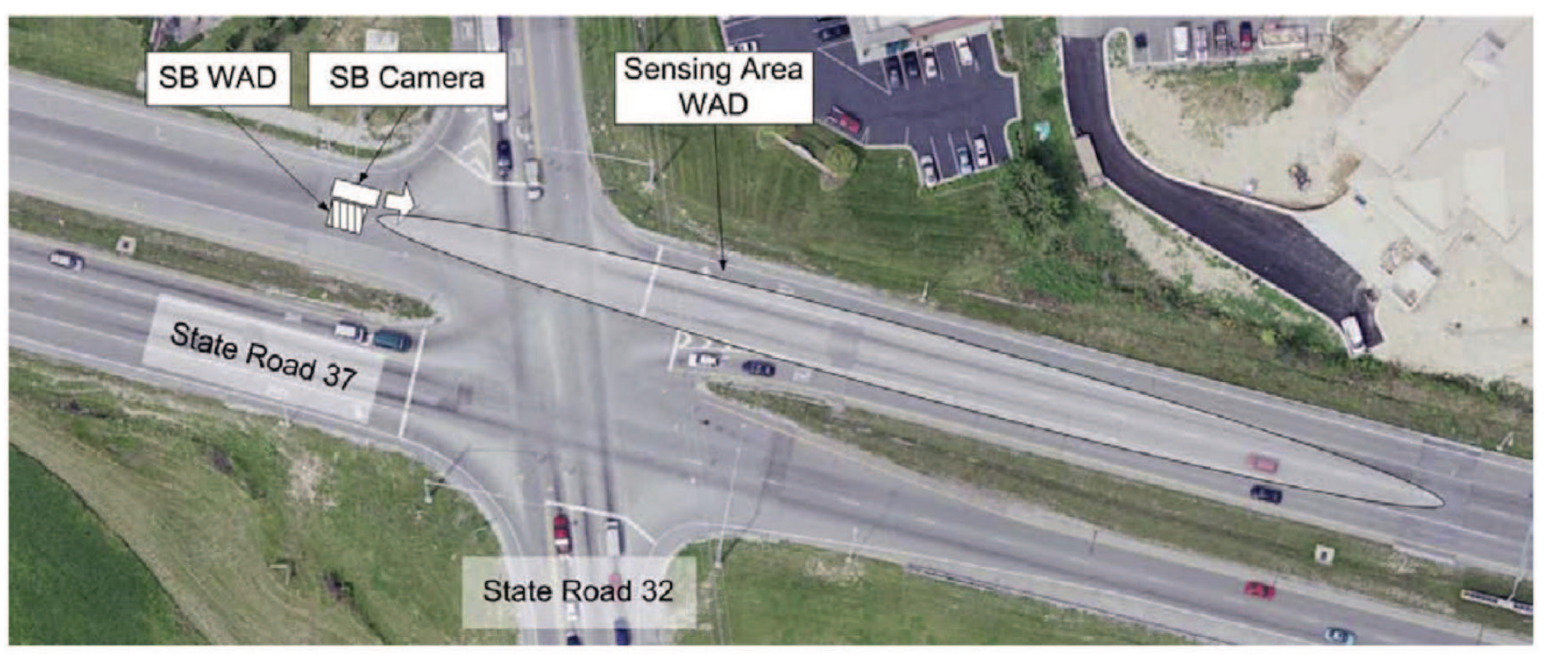

(a) Intersection layout

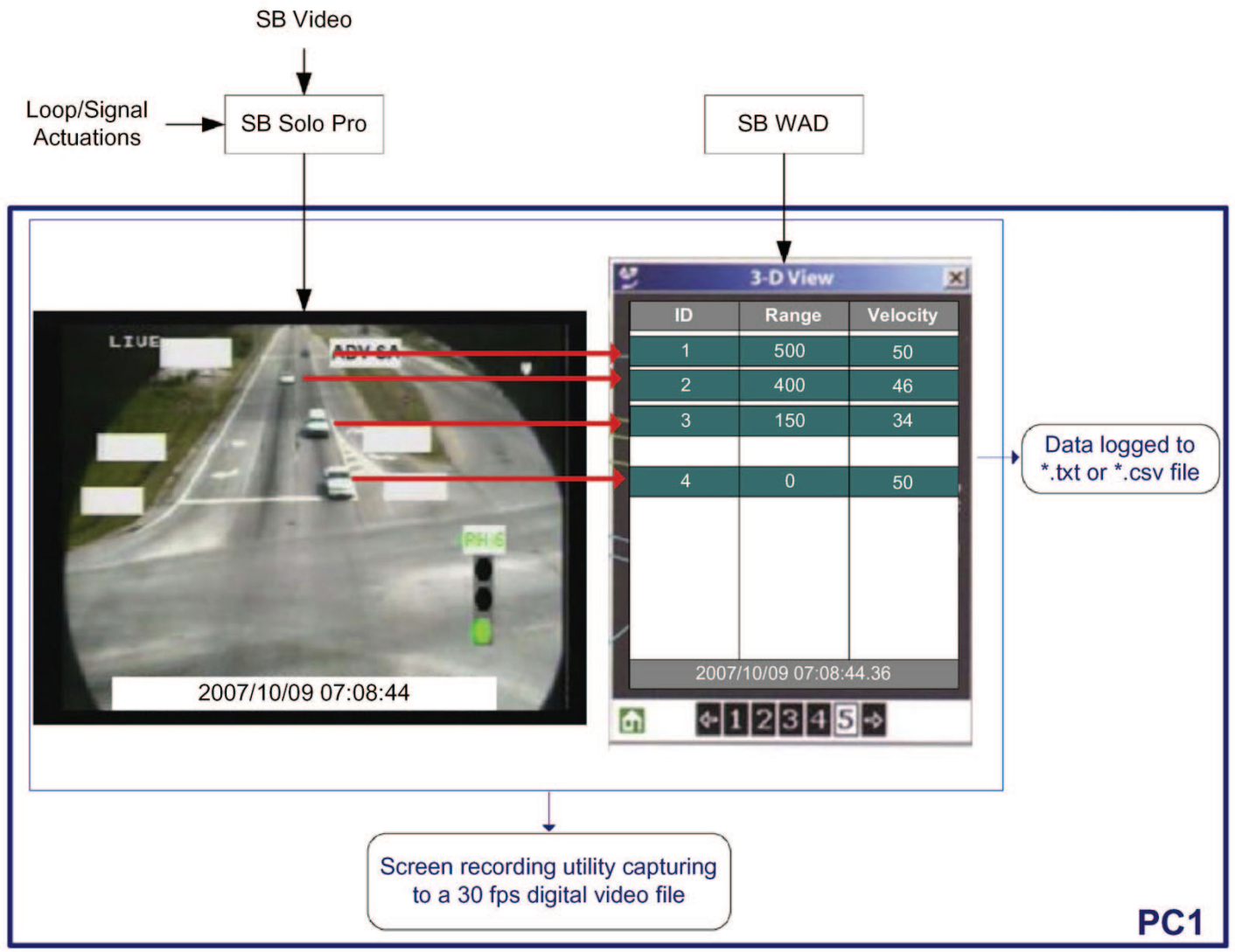

(b) Data collection environment

Figure 3. Data collection at SR 37 @ SR 32 in Noblesville, IN.

massani (1981), a probit model was used to investigate the independent variable instrumental for driver decision for a given intersection. The independent variables tested are listed below.

(1) Distance from the stop bar

(2) Velocity of the vehicle

(3) Time to the stop bar

(4) Deceleration required to stop the vehicle within in the stop bar

(5) Acceleration required by the vehicle to cross the stop bar prior to onset of red 


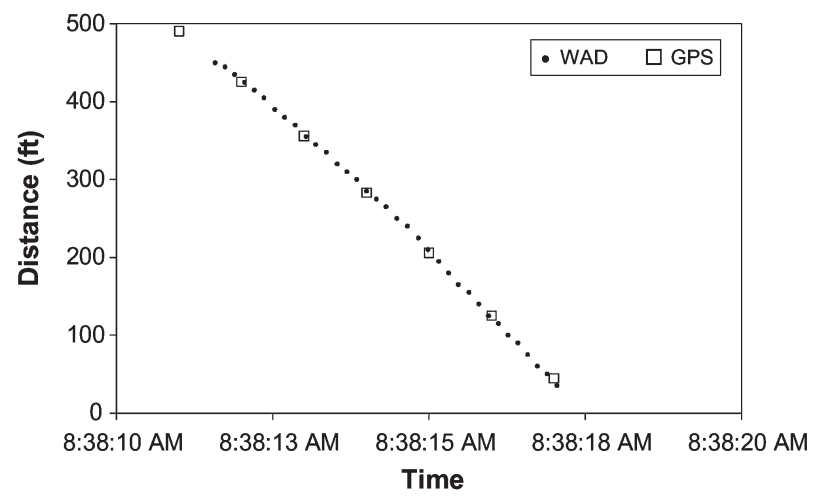

Figure 4. Example comparison between WAD and GPS.

An exhaustive search was performed, on the five variables listed previously, to determine the set of instrumental variables that most significantly explain the variance in driver's choice. Maximum likelihood estimation technique was used to obtain estimates of the parameters using LIMDEP (Greene, 1995). Table 1 contains a set of example iterations for the search of best specified model. The final model is shown in Table $1 \mathrm{~d}$.

Acceleration was found to be the instrumental variable used for the decision process by the drivers. Contradictory to the general notion, required deceleration was not found to be a statistically significant variable, which can be explained as follows. Typically, a vehicle can decelerate at a higher rate than it can accelerate at a specific velocity. Also, the power to accelerate reduces at higher velocities whereas deceleration rates remain constant for varying vehicle velocity for the same pavement and weather conditions. Human tolerance of acceleration or deceleration will be the same in terms of magnitude. Therefore, a driver associates more risk with acceleration due to the car design. Required acceleration subsequently becomes the significant variable for the decision to stop and go. The range of acceleration or deceleration to be used at these distances lies in the comfort ranges of human body tolerance, but the availability of acceleration is limited by the car design.

Table 1. Example iterations to get best fit probit model for stop and go decisions.

(a) Model with constant term, deceleration and acceleration

Number of observations: 2870

Unrestricted log likelihood: -537.2913

Restricted log likelihood: -1536.646

$\operatorname{Prob}\left[\chi^{2}>659.5153\right]=0.00$

$\mathrm{AIC}=.37651$

Variable name

Value

T-stats

Constant

0.172

0.556

Deceleration

$-0.187$

$-0.65$

Required acceleration

0.148

11.846

(b) Model with constant term and time

Number of observations: 2870

Unrestricted log likelihood: -540.9252

Restricted log likelihood: -1536.646

$\operatorname{Prob}\left[\chi^{2}>659.5153\right]=0.00$

$\mathrm{AIC}=.37835$

\begin{tabular}{lrr} 
Variable name & Value & T-stats \\
\hline Constant & -6.35 & -24.806 \\
Time to Stop Bar & 1.36 & 24.405 \\
\hline
\end{tabular}

(c) Model with acceleration (final model)

Number of observations: 2870

Unrestricted log likelihood: -537.5157

Restricted log likelihood: $\mathbf{- 1 5 3 6 . 6 4 6}$

$\operatorname{Prob}\left[x^{2}>659.5153\right]=0.00$

$\mathrm{AIC}=.37597$

\begin{tabular}{lrr} 
Variable name & Value & $T$-stats \\
\hline Constant & -0.027 & -0.626 \\
Required acceleration & 0.15 & 24.846 \\
\hline
\end{tabular}




\subsection{Test for driver homogeneity}

Mixed logit models were investigated in order to determine the homogeneity of drivers at an intersection. Mixed logit is a highly flexible model that can approximate any random utility model. It precludes the three limitations of standard logit by allowing for random taste variation, unrestricted substitution patterns, and correlation in unobserved factors over time (McFadden and Train, 2000). Mixed logit model assumes a random variation in the parameters across the observations, implying that if the driver's perception of the required acceleration and the driver's threshold of comfortable acceleration vary over the population, it can be captured through mixed logit models. No statistically significant standard deviation of the parameter distribution was found for the constant or the required acceleration implying that the set of driver at the intersection of SR37 and SR32 in Noblesville, Indiana, is significantly homogeneous.

\subsection{Sample size check}

A series of validation tests were performed using different sizes of training samples from the data to check that the number of observations were sufficient to develop a robust model.

Two types of error were defined: 1-0 and 0-1. The number one was assigned to vehicles proceeding through the intersection, while a value of zero was assigned to vehicles that stopped. The first term (1-0 or $0-1)$ corresponds to the observed value and the second term corresponds to the predicted value by the model.

- Error: The actual vehicle proceeds through the intersection; however, the model predicts the vehicle stopping.

- Error: The actual vehicle stops at the intersection, while the model predicts it to proceed through the intersection.

The total data set (2870) was randomly divided into two data sets: Training and Validation. The size of training data set was varied from 50 observations to 2,350 observations and the size of validation data set was kept constant at 500 observations. Training data sets were used to fit a probit model for predicting probability of stopping. The model developed was used predict the driver's decision in the validation data set. The 1-0 and 0-1 errors in prediction of validation data were computed. For each size of training data $(50-2,350)$ the process was repeated 1,000 times.

Figure $5 \mathrm{a}$ and $\mathrm{b}$ show that the 95th and 5th percentile errors converge as the number of observations sampled increases. For our data set it should be noted that both the 1-0 and 0-1 errors are less than 5\%. Hence, 2,870 observations are sufficient for modeling probability of stopping with a high degree of accuracy.

\section{Underlying theory of driver's decision}

Driver behavior at the onset of yellow is essentially a binary choice process, where the driver decides to stop or go. Let $A_{p}$ be the acceleration perceived by a randomly chosen driver from the population. Since the perception $A_{p}$ would vary for a driver based on several independent factors, such as perception of the distance from the stop bar, perception of the speed at that moment, perception of the yellow interval based on past experience etc., we can model $A p$ as a normally distributed random variable as shown in Figure 6.

$$
A_{p}=A_{\text {req }}+\xi
$$

where $A_{r e q}$ is the required acceleration to safely enter the intersection at the end of yellow. The term $\xi$ is a random variable is assumed to be normally distributed. We assume that $\xi \sim N\left(0, \sigma_{\xi}^{2}\right)$. Also, if $A_{p}$ is greater than a threshold of comfortable acceleration $A_{t}$, the drivers decide to stop otherwise they decide to go. It should be noted that we are assuming a fixed comfortable acceleration threshold over the driver population as the homogeneity in driver population has been shown through data analysis. The probability of stopping can then be calculated as

$$
\begin{aligned}
& P_{\text {STOP }}=\operatorname{Pr}\left(A_{p}>A_{t}\right) \\
& P_{\text {STOP }}=\operatorname{Pr}\left(z<\frac{\left(A_{r e q}-A_{t}\right)}{\sigma_{\xi}}\right)=\Phi\left(\frac{A_{r e q}-A_{t}}{\sigma_{\xi}}\right)=\Phi\left(a \times A_{\text {req }}+b\right)
\end{aligned}
$$

where $\Phi(\bullet)$ denotes the standard normal cumulative normal function and the functional form $\Phi\left(a \times A_{\text {req }}+b\right)$ is a probit construct. Also, $a=1 / \sigma_{\xi}$ and $b=A_{t} / \sigma_{\xi}$.

Table 1c show the values of parameters $a$ and $b$ estimated for the probit model in Equation (10).

\section{Driver's detected error resulting in minor and severe traffic conflicts}

The driver decision process at the onset of yellow is a dynamic feedback system. The consequences of any decision made by the driver at a certain time step are fed back to him in the next time step (as shown in Figure 2). If a driver erroneously decides to go, even though the required acceleration is greater than the threshold, the driver would realize it after some duration of time. Similarly, an error would occur if a driver decides to stop even though the acceleration 


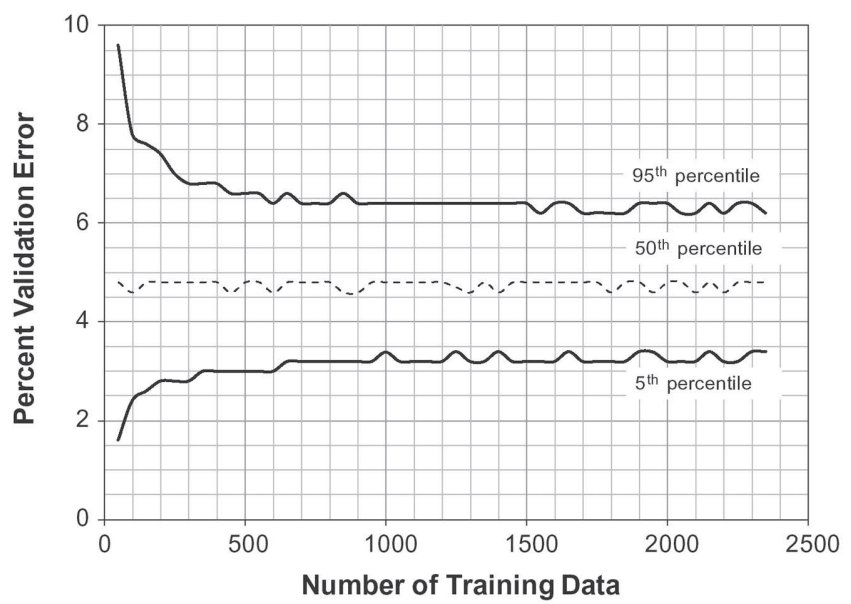

(a) Model predicts the vehicle to stop when it actually went (1-0 error)

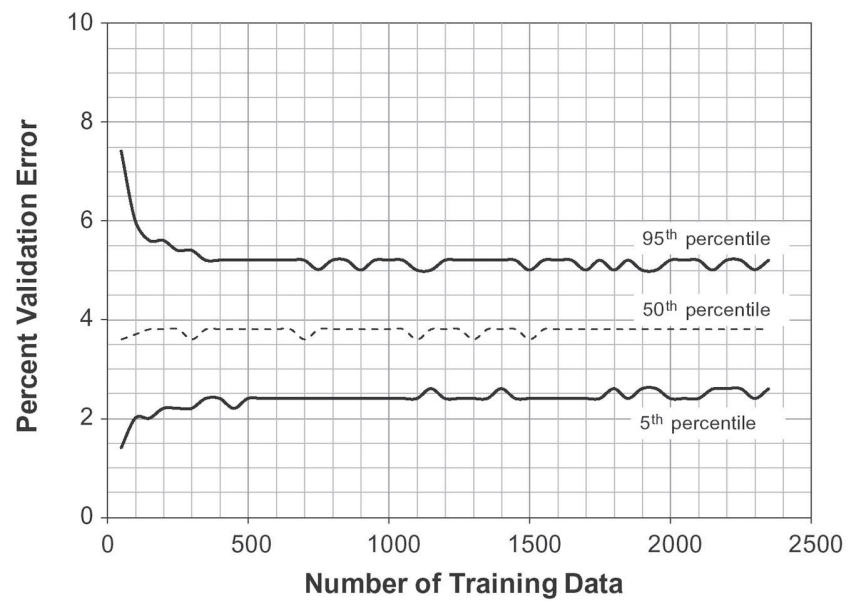

(b) Model predicts the vehicle to go when it actually stopped (0-1 error)

Figure 5. Validation error limits with increasing training data.

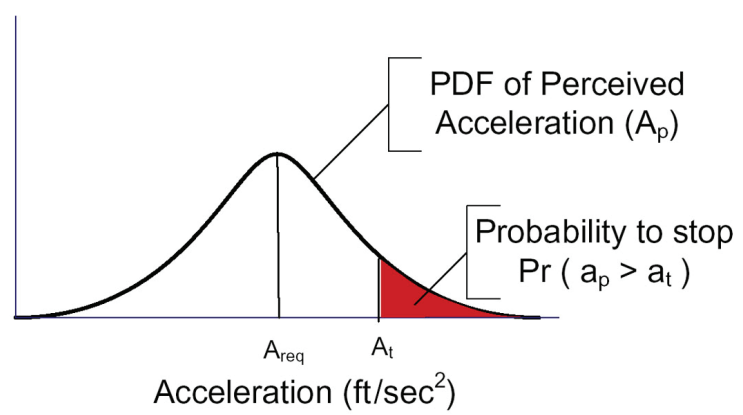

$A_{\text {req }}:$ Required acceleration

$A_{t}: \quad$ Comfortable acceleration threshold

Figure 6. Probability density function for perceived acceleration.

required is less than the comfortable acceleration threshold. The probability of a traffic conflict, therefore, will be the same as the probability of having a detected error (as shown in Figure 6).

$$
P_{\text {CONFLICT }}= \begin{cases}P_{\text {STOP }} & \forall A_{\text {req }}<A_{t} \\ P_{\text {GO }}=1-P_{\text {STOP }} & \forall A_{\text {req }}>A_{t}\end{cases}
$$




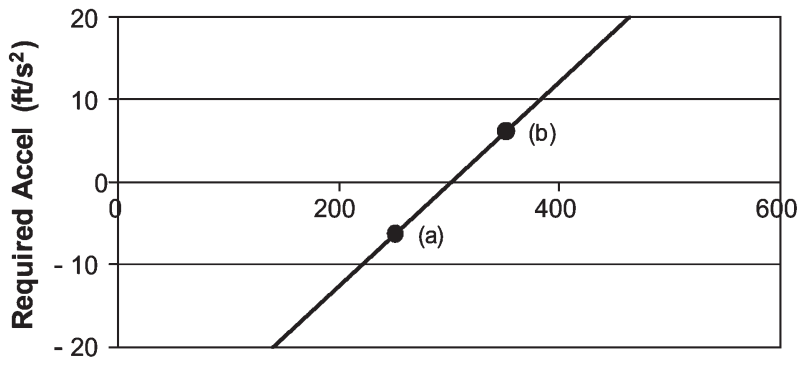

(a) Required acceleration to cross stop bar by the end of yellow phase for a vehicle at $45 \mathrm{mph}$

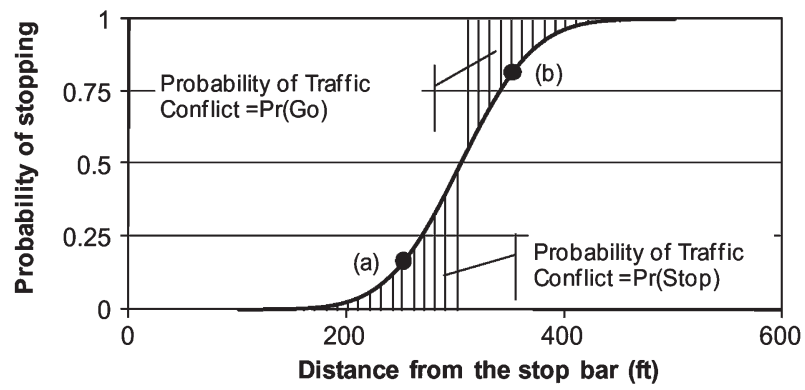

(b) Probit model for driver's stop decision probability for vehicle at $45 \mathrm{mph}$

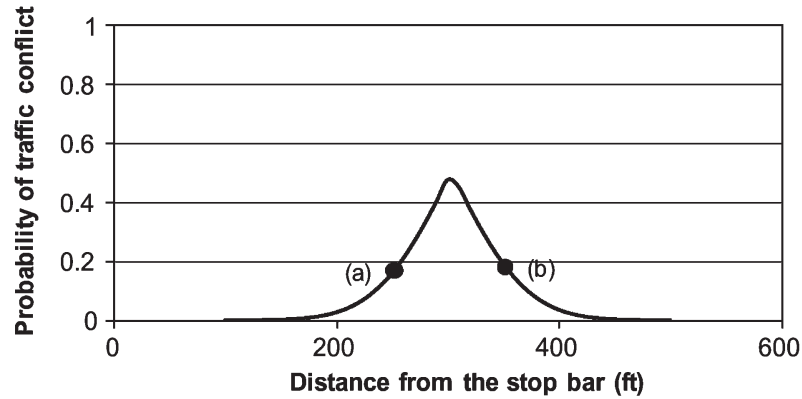

(b) Dilemma hazard function for vehicle at $45 \mathrm{mph}$

Figure 7. Underlying theory of traffic conflict.

Figure 7a and b show that, for a given velocity, the probability of stopping can be obtained by using probit model shown in Equation (10). For example, for points $a$ ( $252 \mathrm{ft}$ from stop bar) and $b$ ( $352 \mathrm{ft}$ from stop bar) on the approach, the probability of stopping for a car travelling at $45 \mathrm{mph}$ is equal to 0.2 and 0.8 , respectively. Figure $7 \mathrm{c}$ presents the probability of traffic conflict function for a vehicle travelling at $45 \mathrm{mph}$ (assuming a yellow phase of $5 \mathrm{~s}$ ). In this figure, at point $a$ (252 ft from stop bar) a vehicle driving at $45 \mathrm{mph}$ can comfortably cross the stop bar without acceleration (assuming an yellow phased duration of $5 \mathrm{~s}$ ). So, any vehicle stopping at this point will face a traffic conflict. Therefore the probability of conflict at point $a$ for vehicles travelling at velocity of $45 \mathrm{mph}$ would be equal to the probability of stopping (0.2). Similarly, for point $b$ (352 ft from stop bar) any vehicle attempting to cross would face a conflict and the probability of perceived conflict at this point for vehicles travelling at $45 \mathrm{mph}$ would be equal to probability of going $(1-0.8=0.2)$. Hence the probability of traffic conflict at both points $a$ and $b$ is 0.2 . Figure $7 \mathrm{c}$ shows the dilemma hazard function for a vehicle travelling at $45 \mathrm{mph}$.

\section{Application of dilemma hazard function using existing controllers}

The optimal operation of intersection demands that the total cost levied on all the drivers should be minimized. The total system cost can only be minimized if the marginal benefits of extending the main-street green (beyond the minimum green time) to reduce the probability of traffic conflict is greater than the marginal increase in cost of delay on the accumulated queue of the opposing movement. Since, the queue length on the side streets will increase with the passage of the main-street green, the protection provided to the main street driver has to be dynamically reduced. 
This section will present a methodology for implementing dynamic dilemma zone protection. For the purpose of illustration we determine the size of the zone of protection for a single vehicle moving at $45 \mathrm{mph}$ with the passage of main-street green. We assume that the speed of the vehicle and the lane information is known. In field, this information can be obtained using a speed trap. Note that the algorithm proposed is only used to reduce the protection provided for single vehicle conflict, full protection will be provided if there are more than two vehicles in their dilemma hazard zone in same lane.

\subsection{Cost of delay associated with extension of green phase of the high speed approach}

The cost of extending the green for clearing the vehicle from the high risk zone can be calculated by using the amount of delay incurred by the queue formed on the stopped phases. Figure 8 illustrates the concept of increase in delay for extending a through green by a single vehicle extension $\left(t_{\text {ext }}\right)$. The un-shaded queue polygon in the Figure 8 is the delay experienced by the vehicles in opposing movement if the green were terminated without the green extension. The extra delay is shown as the shaded area and this extra delay accrues to the side street if the through phase is extended by a time equal to $t_{\text {ext }}$. The extra delay associated with extending the main-street green phase by $t_{\text {ext }}$ after $r$ seconds of green has elapsed is given by Equation (12).

$$
\Delta \text { Delay }=\frac{q_{o p p}}{\left(1-\frac{q_{o p p}}{s_{o p p}}\right)} \times r \times t_{\text {ext }}+\frac{q_{o p p}}{2 \times\left(1-\frac{q_{o p p}}{s_{o p p}}\right)} \times t_{\text {ext }}^{2}
$$

where $\Delta$ Delay, is the increase in the total delay for extending through green by a unit vehicle extension (veh-sec); $q_{\text {opp }}$ the total volume in the opposing direction (veh/s); $s_{o p p}$, the saturation flow rate for the opposing movements (veh/s); $r$, the red time elapsed for the opposing movements (sec); and $t_{\text {ext }}$ is the vehicle extension time (sec).

The increase in the total system delay is multiplied by the cost of delay ( $\$ / \mathrm{veh} / \mathrm{s})$ to obtain the cost of extending the high speed through phase by a unit vehicle extension. For illustration purposes in this paper we use mean hourly rate income for United States 20.32 \$ $/ \mathrm{hr}$.

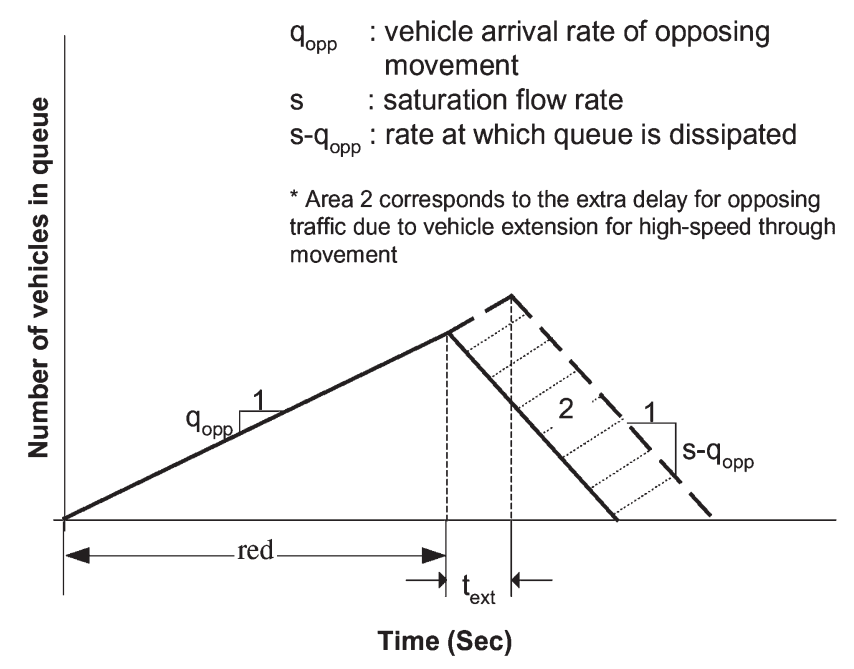

Figure 8. Increase in delay of the standing queue due to vehicle extension.

Table 2. Estimation of cost associated with a traffic conflict.

\begin{tabular}{|c|c|c|c|}
\hline Type of crash & $\begin{array}{l}\text { Cost estimate for motor vehicle crashes } \\
\text { National Safety Council (2007b) }\end{array}$ & $\begin{array}{l}\text { Ratio of each } \\
\text { type of crash }\end{array}$ & Ratio * Cost \\
\hline Death & $\$ 1,130,000$ & 1 & $\$ 1,130,000$ \\
\hline Nonfatal disabling injury & $\$ 61,600$ & 53 & $\$ 3,264,800$ \\
\hline Property Damage only & $\$ 7,500$ & 209 & $\$ 1,567,500$ \\
\hline Weighted average cost per crash $[\operatorname{Cost}(\$ / \mathrm{Crash})]$ & & & $\$ 22,670$ \\
\hline $\begin{array}{l}\text { Probability of getting involved in a crash given a traffic } \\
\text { conflict }[\operatorname{Pr}(\operatorname{Crash} \mid T C)] \text { Gettman et al. (2008) }\end{array}$ & & & 0.00005 \\
\hline $\begin{array}{l}\text { Estimated benefits of preventing a traffic conflict } \\
{[\text { Benefits }(\$ / T C)=\operatorname{Cost}(\$ / \text { Crash }) \times \operatorname{Pr}(\text { Crash } \mid T C)]}\end{array}$ & & & $\$ 1.13$ \\
\hline
\end{tabular}




\subsection{Safety benefits}

The dollar value of safety benefits of extending the green interval can be obtained by multiplying expected probability of having conflict with the cost of conflict. Table 2 illustrates an example of calculating the benefits of preventing a single vehicle traffic conflict. Columns 1 and 2 in Table 2 list the type of crashes and the cost associated with them, respectively, as reported by the National Safety Council (2007b). The weighted average cost of the accident is calculated using the ratios of the type of accidents. The estimated benefits of preventing traffic conflict are obtained as the product of the average accident cost and the probability of occurrence of a crash given a traffic conflict has occurred (Gettman et al., 2008). Based on this methodology, the estimated benefit of preventing a single traffic conflict is $\$ 1.13$. Alternatively, the number of crashes occurring at the intersection and corresponding conflicts can be observed for a period of time. The ratio of the number of crashes to the number of conflicts can be used as the probability of a crash given that the conflict occurred.

\subsection{Calculation dynamic dilemma protection boundaries}

We assume that traffic signal controller has a resolution of $0.1 \mathrm{~s}$. An initial dynamic protection region is defined and with the passage of main-street green this region is reduced with steps of $0.2 \mathrm{~s}$. The step size of 0.2 is chosen because of the controller resolution and to maintain symmetry such that probability of conflict at the start and end of dilemma zone boundary is the same. Dynamic dilemma zone boundaries (DDZB) of protection at different duration of green can be calculated using the following step.

(1) The initial DDZB is the region where the probability of having a traffic conflict is greater than 0.1 . This can be calculated using dilemma hazard function as shown in Figure 9 . For $45 \mathrm{mph}$, the protection area is $376 \mathrm{ft}(5.7 \mathrm{~s})$ - $238 \mathrm{ft}(3.6 \mathrm{~s})$ from the stop bar. It should be noted that the decision of starting dynamic dilemma zone boundary depends on the site engineer, the value of 0.1 is chosen for the purpose of illustration.

(2) The next step is to calculate the expected probability of traffic conflict for the dynamic protection region. The expected value of probability of traffic conflict can be calculated using Equation (13). Figure 9 shows expected probability of traffic conflict calculated for two DDZB (3.6-5.7 s and 4.0-5.3 s).

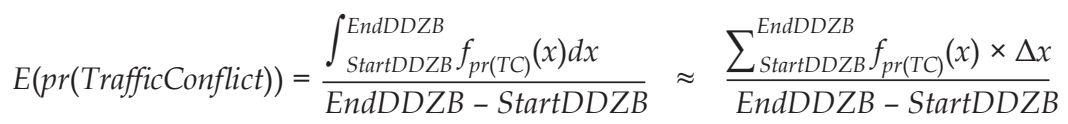

where $E(\operatorname{pr}($ Traffic Conflict $))$, is the expected probability of having traffic conflicts over the given dynamic dilemma zone region; StartDDLB, the start dynamic dilemma zone boundary distance; EndDDLB, the end dynamic dilemma zone boundary distance; and $f_{\operatorname{pr}(T C)}(x)$, is the dilemma hazard function.

(3) The safety benefits of protection zone can be obtained by multiplying expected probability of traffic conflict for a given set of DDZB and multiplying it with the Dollar benefits of saving a single vehicle conflict as shown in Equation (14).

Safety_Benefits $=E(\operatorname{pr}($ TrafficConflict $)) \times$ Dollar Benefits $(\$ /$ Traffic conflict $)$

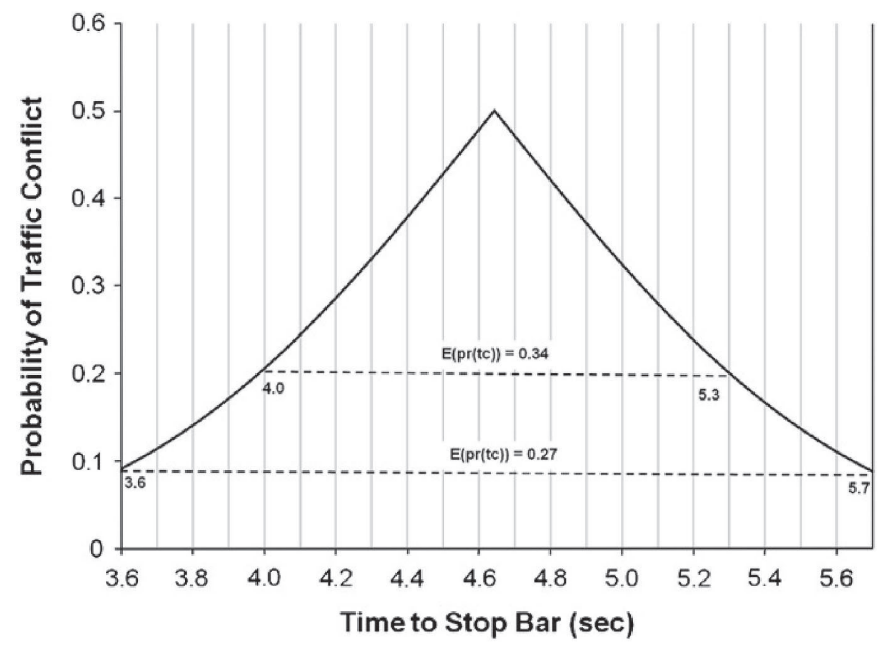

Figure 9. Time based dilemma hazard function for a vehicle travelling at $45 \mathrm{mph}$. 


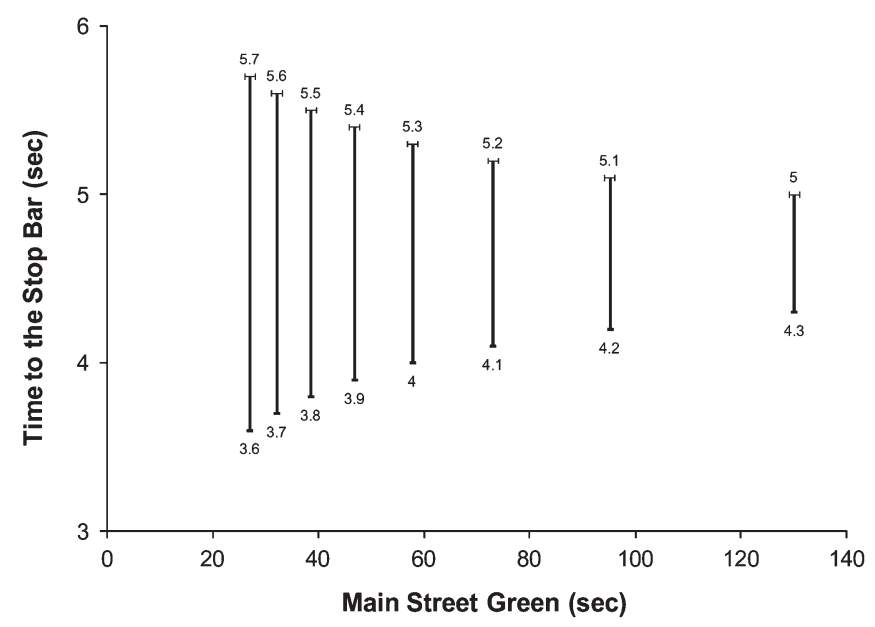

(a) Break-even points for opposing volume of $2500 \mathrm{vph}$

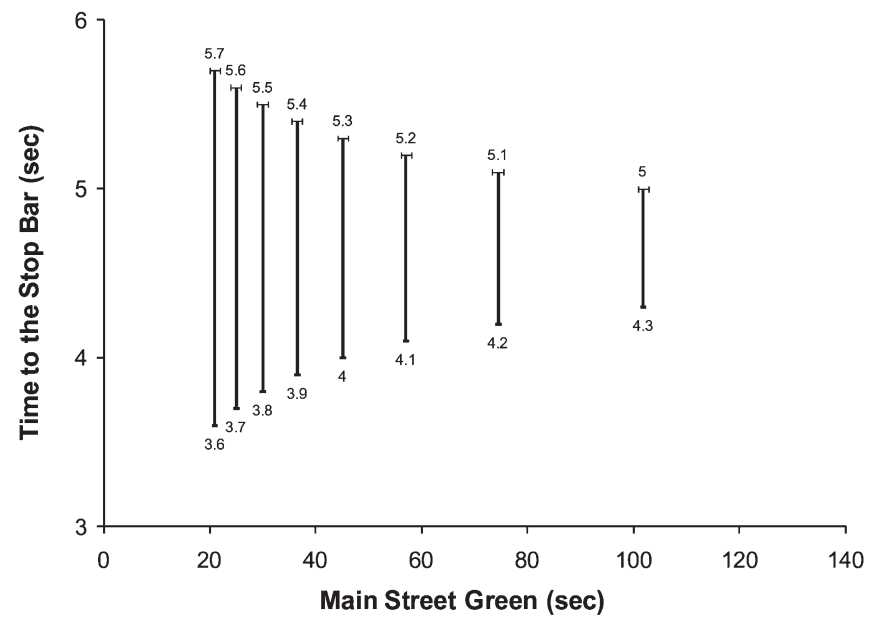

(a) Break-even points for opposing volume of $3500 \mathrm{vph}$

Figure 10. Dynamic dilemma zone boundaries for a vehicle travelling at $45 \mathrm{mph}$.

(4) Break-even points are obtained for deciding the duration until which a specific set of DDZB are used. The break-even points can calculate using Equation (15). Here $t_{i, e x t}$ is the extension time for the $i$ th set of DDZB. For the initial case in our example $t_{1, \text { ext }}$ will be, $5.7-3.6=2.1 \mathrm{~s}$. The term $r_{i}$ represents the break even time till which the $i$ th set of DDZB are used.

$$
\begin{aligned}
\text { SafetyBenefits } & =\text { DelayCosts } E(\operatorname{pr}(\text { TrafficConflict })) \times \text { DollarBenefits(\$/TrafficConflict) } \\
& =\frac{q_{o p p}}{\left(1-\frac{q_{o p p}}{s_{o p p}}\right)} \times r \times t_{i, e x t}+\frac{q_{o p p}}{2 \times\left(1-\frac{q_{o p p}}{s_{o p p}}\right)} \times t_{i, e x t}^{2}
\end{aligned}
$$

Figure $10 \mathrm{a}$ and $\mathrm{b}$ presents the comparison of dynamically changing dilemma protection zone boundaries for hypothetical opposing volume of $2500 \mathrm{vph}$ and $3500 \mathrm{vph}$ in 6 lanes. In the first case, the region between $5.7 \mathrm{~s}$ and $3.6 \mathrm{~s}$ to the stop bar will be protected for first $27 \mathrm{~s}$ of green and then the protection will be dropped to 5.6-3.7 s. The protection region gradually keeps on reducing with the passage of main-street green. It should be noted that the probability of gap-out would increase as the extension interval is reduced. So, lesser and lesser cycle will have max-out. From Figure 10b it can be seen for higher opposing volumes, the break event points for each set of DDZB are earlier than for lower opposing volumes. This is because queue will be building at a higher rate in case of higher opposing volume thus same extension time would lead to higher delay cost for the side street.

\section{Conclusions}

The methodology presented in this paper quantitatively assesses the risk of crash for a driver facing a yellow phase on a high-speed intersection. This methodology extends the current literature of determining dilemma zone 
boundaries to ascertain the probability of a traffic conflict for a single vehicle, providing practitioners a valuable tool to control the signal operation to quantitatively compare trade-offs between safety and efficiency at an intersection. The dilemma zone hazard function developed for single vehicle conflicts is not a binary function, as is typical for the current dilemma zone definitions, but rather is a stochastic function which yields the probability of a conflict given the ambient conditions.

This paper also proposes a dynamic dilemma zone protection algorithm for implementing marginal costs and benefits approach. The proposed algorithm gradually decreases the safety net as the queue on the side streets starts building. This approach increases the economic efficiency of operations. In addition, this approach also reduces the probability of max-out and thus increasing the safety at the intersection.

The dilemma hazard function assigns a higher probability of traffic conflict in the central region and the risk deteriorates towards the boundaries. The dilemma hazard function will be a case sensitive function and will also depend on several other variables like geometric conditions, mode of transportation, weather conditions, time of day, driver's aggressiveness. The range of region to be protected will change depending on whether the dilemma hazard function. A steeper dilemma hazard function will reduce the region to be protected thus the initial DDZB would be a lower value. Thus a case sensitive design of dilemma hazard function is needed for safe and efficient operations. Architecture similar to one present in Noblesville (Figure 2) can be used for real-time development of a dilemma zone hazard function for any intersection. A smart sensor can develop the probability of the stopping curves. Note that the process of manual calculation can be replaced by computational logic built into the smart sensor. The probability of stopping curves can then be used to develop a dilemma hazard function. The use of smart sensors will facilitate data collection to account for variables like mode of transportation, weather conditions, time of day, driver's aggressiveness etc.

\section{Acknowledgments}

This work was jointly supported by the National Science Foundation Program under Grant Number CMS-0528225 and JTRP. The contents of this paper reflect the views of the authors, who are responsible for the facts and the accuracy of the data presented herein, and do not necessarily reflect the official views or policies of the sponsoring organizations, nor do the contents constitute a standard, specification, or regulation.

\section{References}

Bonneson et al., 19944 J. A. Bonneson, P. T. McCoy, and B. A. Moen, Traffic detector design and evaluation guidelines. Report No. TRP02-31-93. Nebraska Department of Roads, Lincoln, Nebraska, 1994.

Chang et al., $1985 \varangle$ M. S. Chang, C. J. Messer and A. J. Santiago, Timing traffic signal change intervals based on driver behavior, Transportation Research Record 1027 (1985), pp. 20-30.

Gates et al., $2007 \varangle$ T. J. Gates, D. A. Noyce and L. Laracuente, Analysis of dilemma zone driver behavior at signalized intersections, Transportation Research Record 2030 (2007), pp. 29-39.

Gazis et al., $1960<$ D. Gazis, R. Herman and A. Maradudin, The problem of the yellow signal in traffic flow, Traffic Engineering (July) (1960), pp. 19-26.

Greene, 19954 W. H. Greene, LIMDEP's Version 7. 0 User's Manual. Econometric Software, Inc., Bellport, New York, 1995.

Gettman et al., $2008<$ D. Gettman, P. Lili, T. Sayed, and S. Shelby, Surrogate safety assessment model and validation. Final Report. FHWA-HRT-08-051, 2008.

ITE technical committee, 1974 ITE Technical Committee 18 (P. S. Parsonson, Chairman), Small-area detection at intersection approaches. Traffic Engineering. Institute of Transportation Engineers, pp. 8-17 (February 1974).

May, 1968 A. D. May, Clearance interval at flashing Systems, Highway Research Record 221 (1968), pp. 41-71.

McFadden and Train, $2000<\mathrm{D}$. McFadden and K. Train, Mixed MNL models for discrete response, Journal of Applied Econometrics 15 (5) (2000), pp. 447-470.

National Safety Council, 2007b « National Safety Council, 2007b. Online at http://www.nsc.org/resources/issues/estcost.aspx

Olson and Rothery, 19624 P. O. Olson and R. Rothery, Driver response to yellow phase of traffic signals, Highway Research Board 330 (1962), pp. 40-51.

Papaioannou, 2007 P. Papaioannou, Driver behavior, dilemma zone and safety effects at urban signalized intersections in Greece, Accident Analysis and Prevention 39 (2007), pp. 147-158.

Sharma et al., $2007 \varangle$ A. Sharma, D. Bullock and S. Peeta, Recasting dilemma zone design as a marginal costs- benefits problem, Transportation Research Record 2035 (2007), pp. 88-96.

Sharma et al., 20064 A. Sharma, D. Bullock and S. Peeta, Limitations of simultaneous gap out logic, Transportation Research Record 1978 (2006), pp. 42-48.

Sharma et al., 2008 A. Sharma, M. Harding, B. Giles, D. Bullock, J. Sturdevant, and S. Peeta, Performance requirement and evaluation procedures for advance wide area detector. In: 87th Transportation Research Board Annual Meeting, Transportation Research Board, Washington, DC (January 2008. ).

Sheffi and Mahmassani, 19814 Y. Sheffi and H. Mahmassani, A model of driver behavior at high speed signalized Intersections, Transportation Science 15 (1) (1981), pp. 51-61.

Smaglik et al., $2005<$ E. Smaglik, D. Bullock and T. Urbanik, An evaluation of lane by lane vehicle detection for actuated controllers serving multi-lane approaches, Transportation Research Record 1925 (2005), pp. 123-133.

Webster and Ellson, $1965<$ F. V. Webster and P. B. Ellson, Traffic signals for high speed roads. Roads Research Laboratory. Technical Paper No. 74 (1965).

Zegeer, 1977 C. V. Zegeer, Effectiveness of green-extension systems at high-speed intersections. Research Report 472. Bureau of Highways, Kentucky Department of Transportation, Lexington, Kentucky (May 1977). 